\title{
Assistência à saúde de pacientes com tuberculose no Município de Santa Maria, RS
}

\author{
Health care for patients with tuberculosis at Santa Maria, RS
}

Evelyn Santos de MATOS; Jane Beatriz LIMBERGER

Centro Universitário Franciscano. Rua dos Andradas, 1614, Santa Maria, RS. CEP

97110708. Brasil.E-mail: evelyn_matos_150@yahoo.com.br

\begin{abstract}
The aim of this work was at evaluating the activities related to the pharmaceutical assistance for tuberculosis in Santa Maria, RS, as well as its adequacy to the guidelines from Controlling of Tuberculosis in Brazil Handbook. Strengths and weaknesses from the actions developed of the Division of Tuberculosis throughout a descriptive observational study were identified in this research, from August 2012 to January 2013. It was noticed a meaningful search for the service, which was made by a diagnosis and follow-up of the treatment, using combined doses. Despite the monthly follow-up of the treatment for symptoms and sputum smears, and the adoption of standard procedures in order to reduce dropouts and relapses as the use of incentives, the adherence keeps remaining the biggest challenge. Based on the overview found, it is suggested the implementation of humanized follow-up practices, approaching patients and health professional during the treatment, with the intention of improving the adherence to pharmacotherapy and promoting life quality to the patients.
\end{abstract}

Key Words: Tubeculosis, Drug Therapy, Medication Adherence

RESUMO:

Esta pesquisa teve como intuito a avaliação das atividades de assistência à saúde para tuberculose no município de Santa Maria, RS, e sua adequação às diretrizes preconizadas pelo Manual de Recomendações para o Controle da Tuberculose no Brasil. Foram identificadas as potencialidades e fragilidades das ações desenvolvidas por meio de um estudo observacional descritivo das atividades desenvolvidas pelo Setor de Tuberculose do município, no período de agosto de 2012 a janeiro de 2013. Foi observada uma significativa procura pelo serviço, no qual é feito diagnóstico e acompanhamento do tratamento, utilizando doses combinadas. Apesar do acompanhamento mensal de sintomas e baciloscopias e da adoção de procedimentos padrão para reduzir abandonos e recidivas como o uso de incentivos, a adesão continua sendo o maior desafio. Com base no panorama encontrado, sugere-se a implementação de práticas de acompanhamento humanizado, aproximando pacientes e profissionais de saúde durante o tratamento, com o intuito de melhorar a adesão a farmacoterapia e promover a qualidade de vida dos pacientes

Palavras-Chave: Tuberculose, Farmacoterapia, Adesão à Medicação 


\section{INTRODUÇÃO}

A tuberculose (TB) é uma doença contagiosa grave causada pela bactéria Mycobacterium tuberculosis também conhecida como Bacilo de Koch. Sua transmissão se dá via sistema respiratório por meio de gotículas contendo o agente infeccioso, e seus sintomas mais típicos incluem tosse persistente há mais de duas semanas, febre vespertina, sudorese noturna e escarro (1). O Brasil constitui o único país das Américas dentre os 22 países considerados pela Organização Mundial da Saúde (OMS) como os de maior prevalência de TB, situação que se deve a urbanização descontrolada, co-infecção com o vírus HIV, aumento da pobreza e alcoolismo (2).

Estratégias de controle vêm sendo elaboradas prevendo ampliação do acesso aos serviços de prevenção, promoção, diagnóstico e tratamento da TB, bem como levantamento de indicadores e capacitação de equipes para atenção em saúde por meio do Plano Nacional de Controle da Tuberculose (3). Para que a disseminação da doença seja controlada, faz-se necessário a busca ativa de casos com a finalidade de diagnosticar a doença no seu início e impedir sua transmissão, sendo esta uma estratégia priorizada nos serviços de saúde. Tal atitude deve ser tomada principalmente em populações especiais, que incluem indivíduos privados de liberdade, em situação de rua, indígenas e profissionais da saúde, nos quais há uma maior incidência da doença $(4,5)$.

A partir do diagnóstico da doença é preciso que tratamento seja iniciado em doses combinadas dos fármacos RHZE (Rifampicina, Isoniazida, Pirazinamida, Etambutol) por 2 meses, seguido de RH (Rifampicina, Isoniazida) por 4 meses, totalizando os 6 meses de tratamento preconizado pela OMS, o qual é bastante eficaz desde que seguido corretamente (6).

No entanto, apesar das medidas de controle e de suporte à $\mathrm{TB}$, um agravante ganha destaque: o abandono, que, no Brasil, permanece em um percentual elevado (14\%) nos últimos 20 anos (7). Dentre os motivos para abandonar o tratamento estão a influência familiar, melhora dos sintomas, alcoolismo, drogas, revolta com a doença, motivos econômicos e co-infecção com HIV (8).

Este agravo preocupa principalmente a população privada de liberdade, a qual, geralmente, possui um tratamento irregular e uma deteç̧ão tardia de resistência advindo da atitude de abandonar o tratamento. A alta endemia no sistema prisional pode ser explicada por celas superpopulosas, mal ventiladas, com pouca iluminação, falta de informações sobre o problema e dificuldade de acesso aos serviços de saúde, o que faz com que recidi- vas e episódios de resistência ao antimicrobiano sejam comuns (9). Uma das formas de garantir a adesão no ambiente prisional pode ser representada pela supervisão direta por profissionais da saúde, capazes de reconhecer os pacientes como doentes e evitar que a medicação seja usada como moeda de troca assegurando a continuidade fora deste ambiente (4).

Além destes, o abandono costuma atingir outras populações, como usuários de drogas, etilistas e coinfectados com HIV. Alcoólatras crônicos apresentam maior interação com as drogas utilizadas no tratamento, apresentando manifestações neurológicas e psiquiátricas incluindo distúrbios de comportamento, psicoses e amnésias. Verifica-se que quando em maior vigilância estes pacientes tendem a cooperar e, portanto indica-se o tratamento diretamente observado (TDO), com orientações sobre o aumento do risco de efeitos colaterais (10).

Pacientes co-infectados apresentam em maior proporção sintomas como diminuição de peso, febre, reação inflamatória exacerbada, e maiores manifestações dos efeitos adversos (4). Indivíduos toxicômanos costumam não aceitar a doença e apresentam diversos efeitos adversos provenientes do tratamento, o que contribui para o abandono (10).

A abordagem ao paciente em risco de abandono torna-se uma estratégia fundamental no controle da TB, à medida que reduz as chances de infecção de comunicantes e a disseminação descontrolada da doença. Neste sentido, esta pesquisa se propôs a acompanhar as atividades do Serviço de Controle de Tuberculose no município de Santa Maria por meio da identificação de potencialidades e fragilidades nos processos de trabalho, a fim de traçar estratégias para resolução dos problemas encontrados, especialmente no que tange a adesão ao tratamento farmacológico.

\section{MÉTODO}

Foi realizada uma pesquisa observacional descritiva e transversal, com o objetivo de avaliar a realidade da assistência à saúde vinculada à tuberculose no município de Santa Maria. Foram acompanhadas as ações exercidas junto à Secretaria de Saúde, de agosto de 2012 a janeiro de 2013, no que tange a temática da TB tendo por base o Manual de Recomendações para o Controle da Tuberculose no Brasil (4), identificando potencialidades e fragilidades das seguintes dimensões: Procura de casos; acompanhamento do caso diretamente observado; registro de pacientes em tratamento; registro de requisições de exames; registros de sintomas respiratórios; preenchimento de fichas de notificação; levantamento de indicadores de monitoramento e avaliação de casos 
de TB, registro de notificação de casos TB multirresistentes; e registro de acompanhamento pós-cura. Os dados observados foram anotados em planilha específica e discutidos qualitativamente. Esta pesquisa foi aprovada pelo comitê de ética em pesquisa da Unifra, sob número de registro 420.2011.2.

\section{RESULTADOS E DISCUSSÃO}

Foi constatado, no tempo em que foram observadas as atividades, que o número médio de atendimentos diários era de 10 pacientes. A unidade recebeu encaminhamentos por parte de hospitais, pronto-atendimentos, postos de saúde, além de atender pacientes sintomáticos que buscaram o serviço diretamente.

Foi observado que a vigília da tomada do medicamento diária não era realizada com todos os pacientes e sim somente com aqueles mais reticentes, crianças e idosos. Além disso, eram oferecidos incentivos aos pacientes para que houvesse a continuidade do tratamento, como vale-transporte e cestas-básicas, considerando que grande parte da população afetada tinha condições financeiras precárias.

Os pacientes eram orientados pela enfermeira do local sobre a farmacoterapia e seus efeitos adversos. O acompanhamento do paciente ocorreu em periodicidade mensal por meio do acompanhamento da regressão dos sintomas e resultados das baciloscopias.

Durante o acompanhamento das atividades, foi observado também que dados de sintomáticos respiratórios eram registrados assim que o resultado das baciloscopias era emitido, gerando indicador da prevalência dos casos bacilíferos e incidência da doença no município.

\section{DISCUSSÃO}

Procura de casos. A busca por sintomáticos respiratórios é a estratégia de grande importância na descoberta de casos bacilíferos, ou seja, indivíduos que são capazes de transmitir a doença. Sua abordagem e tratamento permitem a interrupção da transmissibilidade e a incidência da doença (4). É importante salientar que o conhecimento de um caso novo da doença torna possível rastrear os contatos para investigação a fim de impedir que a doença se manifeste nestes $(11,12)$. Foi observado, durante a coleta de dados, que a busca ativa por casos de TB foi também estimulada nas unidades básicas de saúde, unidades de saúde da família e hospitais, por meio de cartazes informativos sobre a doença.

Acompanhamento do caso diretamente observado. Durante o atendimento, o paciente era questionado a respeito da persistência da tosse, sendo então orientado a coletar duas amostras de escarro, uma imediata e outra no dia seguinte, em local apropriado, a fim de ser realizado o teste de baciloscopia. Este procedimento ocorria conforme descrito no Manual de Recomendações para Controle de Tuberculose no Brasil (4). Em caso de confirmação de diagnóstico, o paciente era orientado a iniciar o tratamento imediatamente, sob a observação e acompanhamento de profissionais da saúde durante todo o tratamento.

O Tratamento Diretamente Observado (TDO) era realizado no serviço do município por meio da administração local do medicamento, e destinado principalmente a pacientes que resistiam ao tratamento, como etilistas e portadores do vírus HIV. Esta prática pode ser considerada comum, e este acompanhamento visa a maior adesão e não surgimento de tuberculose multirresistente (13).

Cardoso et al (2012) avaliaram o TDO sob a óptica dos profissionais de saúde envolvidos e confirmou que para algumas localidades a implementação desta prática foi mais difícil por não haver parcerias com os agentes comunitários, articulação entre as equipes de saúde e os próprios incentivos, como vale-refeições e vale-transporte, além da falta de acolhimento adequado dos casos (13).

A equipe multiprofissional tem por objetivo trabalhar no acolhimento e orientação de pacientes portadores de TB, a fim de facilitar a adesão e a conclusão do tratamento destes pacientes. No serviço avaliado, contava-se com um médico infectologista, responsável pelo diagnóstico e acompanhamento do paciente, uma enfermeira, responsável pelo acolhimento e dispensação de medicamentos, e um farmacêutico, incumbido da realização de coletas de materiais e realização dos exames de análises clínicas pertinentes. Foi observado que a presença de um farmacêutico também na dispensação contribuiria para a avaliação faramcoterapêutica e atenção farmacêutica, além promover a busca ativa e resolução de problemas relacionados a medicamentos. Outros profissionais ainda poderiam ser incluídos, tais como psicólogos e assistentes sociais, que contribuiriam para o aprimoramento da acessibilidade e do acesso ao tratamento.

No Rio de Janeiro, entre 2001 e 2006, Cantalice Filho (2009) comprovou que o uso do incentivo alimentício aumentou as taxas de cura e diminuiu o abandono, pois reduziu a interferência de outros fatores socioeconômicos no tratamento (14). Outra ferramenta importante apontada nesta mesma pesquisa refere-se a visitas domiciliares, nas quais se faz possível, além da descoberta de novos casos, o monitoramento da farmacoterapia. Contudo, uma fragilidade das visitas domiciliares consiste na necessidade de reorganização do processo, levando em consideração a vida particular de cada pa- 
ciente. Nestes casos, a responsabilidade pela adesão ao tratamento é compartilhada entre profissionais de saúde e pacientes, não sobrecarregando nenhum dos atores envolvidos (15).

Um estudo realizado em Vitoria - ES, no período de 2003 a 2005, mostrou que dentre os pacientes que escolheram ter um supervisor domiciliar, 99\% obtiveram a cura, negativando as baciloscopias conforme a evolução do tratamento (16).

Registro de pacientes em tratamento. Foi observado que o registro dos pacientes em tratamento farmacológico ocorria no Livro de Registro e Acompanhamento de Tratamento dos Casos de Tuberculose, um instrumento oficial do Ministério da Saúde (4). Nele ficam registrados os dados do paciente, obtidos por meio de anamnese e exames, os quais tornam possível a consolidação dos dados e análise dos casos atendidos no local. O Ministério da Saúde preconiza que os resultados das baciloscopias devem ser imediatamente registrados no instrumento, com o objetivo de servir como indicador epidemiológico e operacional (4).

O Registro de Acompanhamento é a forma mais utilizada para registro de informações sobre o tratamento farmacológico de pacientes com tuberculose. Segundo o estudo de Cavalcanti et al (2012), realizado em 13 municípios do Rio de Janeiro, o instrumento informa o histórico do paciente do início ao fim do tratamento e permite a troca de informações entre os profissionais envolvidos em todos os níveis de atenção, da básica à especializada (17). Este feedback é importante pois, na maioria das vezes, a atenção básica é o primeiro contato com o paciente infectado, e as informações geradas pelo instrumento permitem o planejamento de ações de promoção da saúde e prevenção da doença.

Registro de requisições de exames. $\mathrm{O}$ registro de requisições de exames era realizado por meio do Sistema Gerenciador de Ambiente Laboratorial (GAL), sendo que resultados positivos ou suspeitos eram encaminhados ao Sistema de Informação de Agravos de Notificação (SINAN). Os principais exames realizados durante o acompanhamento de casos de tuberculose eram o exame de cultura para micobactérias e o isolado bacteriano que é um teste de sensibilidade realizado em pacientes com tuberculose extra-pulmonar, para que se identifique a espécie da bactéria por meio de técnicas como histopatologia, produção de enzimas e a própria cultura. Estes procedimentos estão preconizados na Nota Técnica Conjunta $n^{\circ}$ 02/2011, do Centro Estadual de Vigilância em Saúde do Rio Grande do Sul (18).

Preenchimento de fichas de notificação. $O$ SINAN é a fonte de dados mais confiável e a melhor ferramenta para vigilância epidemiológica em todas as esferas de atenção, uma vez que dissemina os dados e informações de maneira ágil e eficiente. Para tanto, os registros devem ser realizados com o máximo cuidado, uma vez que só podem ser atualizados ou corrigidos pelo município que está notificando (4). A obtenção de dados com qualidade perpassa o controle das diversas etapas realizadas, desde o registro dos casos na unidade até sua digitação no SINAN, tendo como um dos cuidados mais relevantes a eliminação de duplicidade de dados. No Setor em estudo, esta atualização era feita com periodicidade semanal pela enfermeira responsável.

Registros de sintomas respiratórios. Os pacientes sintomáticos respiratórios também eram notificados para se ter o controle de casos positivos, sendo documentado o nome do paciente e resultados das suas bacilospias com a finalidade de identificar os casos e iniciar a farmacoterapia imediatamente, além de subsidiar o alcance das metas anuais de tratamento.

Levantamento de indicadores de monitoramento e avaliação de casos de TB. Para monitorar o tratamento devem ser utilizados dados de todos os exames realizados, número de contatos, TDO, a situação do tratamento e o seu encerramento resultados estes que auxiliam a avaliação de resultados o alcance de metas estabelecidas de atendimento (4).

Esta avaliação torna possível a identificação de casos de tuberculose multirresistente, cujas notificações são encaminhadas para a unidade de referência para validação e envio dos medicamentos específicos para a unidade de saúde a fim de dar continuidade ao tratamento. Além disso, é preconizado o acompanhamento do caso por cinco anos para detectar possíveis recidivas (4). No estudo, foi percebida a preocupação da equipe com o levantamento de indicadores, para que sirvam de subsídios para a orientação de ações que permitam traçar um diagnóstico preciso dos casos de tuberculose no município.

Registro de notificação de casos TB multirresistentes (TB-MDR). Após a confirmação de resistência a Rifampicina e Isoniazida, por meio de cultura e teste de sensibilidade, os casos devem ser encaminhados às unidades de referência para o preenchimento de ficha de notificação, cuja validação permite o envio de medicamentos adequados ao caso. Devem ser solicitadas culturas a cada dois meses e radiografia a cada três meses para acompanhamento (4).

Um estudo realizado na África do Sul, no período de 2003 a 2006, sugeriu que medidas de controle como diagnóstico em tempo correto e rastreamento de casos ativos foram ineficazes para o controle da tuberculose resistente, além de sofrer influência do diagnóstico tardio e tratamento inadequado (19). Esta situação exige que medidas locais sejam tomadas, como rastreamento de casos ativos e estudos de utilização de medicamen- 
tos, visando instituir posologias e regimes de tratamento ideais.

Durante a coleta de dados não foram evidenciados casos de tuberculose multirresistente no município de Santa Maria, apesar do crescente número de casos da doença (Figura 1). Este resultado mostra que as ações vinculadas ao tratamento farmacológico dos pacientes têm sido efetivas, uma vez que a taxa encontra-se abaixo dos valores máximos preconizados para países em desenvolvimento (15\%) (20).

No Brasil, é observado baixo percentual de resistência, ou seja, da forma Tuberculose Multidrogarresistente (MR), considerando que em 2008, o número de casos estimados de TBMR, no mundo, foi de 440.000 (22). O Brasil, em 2009, notificou 411 casos novos de TBMR. (22) No mundo existem focos extremamente preocupantes de TB multidrogarresistente primária na Estônia (14\%); e na Rússia percentuais próximos a 10\% (23). Bacilos resistentes a rifampicina e isoniazida associam-se à maior falha terapêutica, ao pior prognóstico, a mais efeitos colaterais pelo uso de esquemas alternativos e, assim, ao maior custo agregado (24).

Figura 1: Distribuição do número de casos notificados de Tuberculose, curas e abandonos, segundo a área de abrangência da Secretaria Municipal de Saúde de Santa Maria- RS, 2007-2011. Fonte: SINAM/MS, 2013. (21).

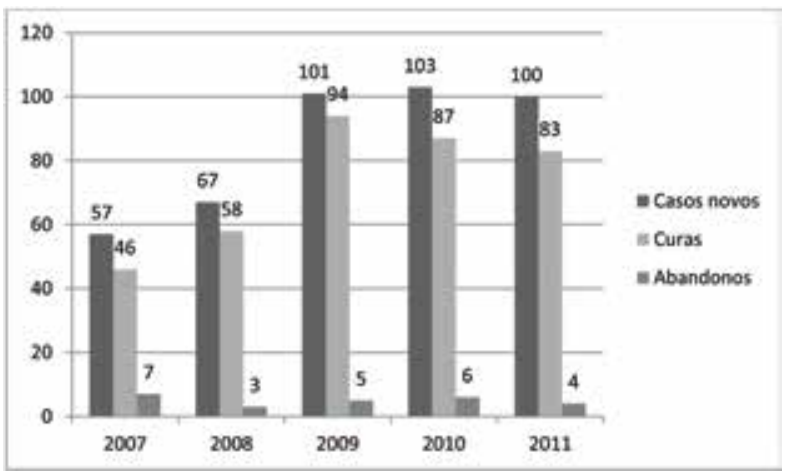

Registro de acompanhamento pós-cura. Após a cura é recomendado o acompanhamento dos casos pelo menos por mais cinco anos, com frequência quadrimestral nos primeiros dois anos e semestral nos três anos seguintes. O acompanhamento se dá por meio do acompanhamento de sintomas, baciloscopias, radiografias e cultura (4). Contudo, no setor de tuberculose local, o acompanhamento era dificultado principalmente por falta de pessoal suficiente para acompanhar cada caso, dificuldade de deslocamento até unidades básicas de saúde.

Fragilidades e perspectivas para adesão à terapêutica. Apesar de todos os esforços e registros para que se consiga uma adesão adequada e se evite casos de resistência, o abandono segue como um problema importante, como se é mostrado na Figura 1 (21).

Vários são os motivos que fazem com que o abandono ocorra, e dentre eles destacam-se questões inerentes ao próprio paciente, do tratamento e dos profissionais de saúde. Estudos realizados em Manaus (AM), Fortaleza (CE) e em João Pessoa (PB) apontaram que a falta de adesão se deveu a falta de informação, representações negativas da enfermidade, etilismo, tabagismo, drogas ilícitas, problemas socioeconômicos, grande quantidade de comprimidos a serem administrados e a crença na cura pela fé $(25,26)$.

Por conseguinte, algumas estratégias foram criadas para diminuir a incidência de abandono, visando à conclusão do tratamento. A revisão sistemática de Jmachoki, Kredo e Volmink (2012) avaliou a melhoria da adesão por meio de diferentes estratégias como telefonemas e visitas domiciliares, efetivas no aumento do sucesso da farmacoterapia com crianças (27). Como o TDO pode ser considerado invasivo por alguns pacientes e limitado para aqueles que vivem perto das unidades de saúde, Garfein et al. (2012) desenvolveram um estudo-piloto usando celular para fazer registro de vídeos dos pacientes administrando seu medicamento (28). Este método foi aplicado em San Diego, Califórnia (EUA) e Tijuana (México) e mostrou que grande parte dos vídeos foi recebida na hora marcada e assistida por um profissional da respectiva unidade. Apesar de tratar-se de uma atenção que demanda elevados custos, seus resultados podem ser aplicados de maneira promissora tanto para TB, quanto para outras doenças que necessitem de maior atenção.

Um estudo realizado na África do Sul em 2002 apontou que a abordagem ao paciente deve ser centrada no indivíduo e que o respeito entre ele e prestadores de serviço deve ser sadia, para garantir adesão (29). Mesmo que o país sofra com a endemia de co-infecção com o vírus HIV, vem investindo em novas políticas de saúde e melhorando a motivação pessoal de quem trabalha com esses pacientes.

Na Etiópia, 44 unidades de saúde foram avaliadas e foi observado que se os prestadores de cuidado tivessem treinamento inadequado frente à doença, a tendência era de interrupção do tratamento, sugerindo que a formação de quem cuida deve ser aprimorada a fim de melhorar afinidade entre profissional e paciente e estimular a adesão (30). Nesta mesma linha, um estudo realizado na Tanzânia, concluiu que o paciente desejar ser ajudado e ter disponibilidade de comunicação com os profissionais contribui para a adesão ao tratamento (31).

Nota-se que a maioria das intervenções propostas tem em comum a implementação de práticas de humanização em saúde, na qual se pretende conhecer, enten- 
der, comunicar, conversar com quem precisa de cuidados, tratando pacientes como iguais (32). No Brasil, a humanização em saúde tem status de política pública, que prevê a criação de espaços para que haja troca de conhecimentos e trabalho em equipe, levando em consideração as diversas opiniões na construção de políticas (33). Assim, para que seja obtido sucesso no tratamento da tuberculose, torna-se fundamental o processo de humanização de práticas de cuidado, visando a elaboração conjunta de estratégias para a adesão, partilhando compromissos entre doente e prestador de serviço (26).

\section{CONCLUSÃO:}

A pesquisa permitiu verificar a situação em que se encontra o setor de Tuberculose da região de Santa Maria, identificando suas potencialidades e fragilidades, principalmente no que tange à assistência à saúde do paciente. Foi observado que existem déficits de registros, e apesar das orientações dadas a respeito do tratamento aos pacientes serem claras, a adesão continua sendo o principal problema. Como os incentivos não estão dando sendo efetivos, estudos sugerem que a implementação de práticas de humanização em saúde permitiriam o contato mais próximo entre paciente e profissional de saúde por meio de telefonemas, visitas ao domicílio, maior veiculação de informações, além dos próprios incentivos.

Essas medidas certamente trariam benefícios, não somente aos usuários do Programa de Tuberculose, mas também aos seus familiares e à sociedade, uma vez que a adesão à terapêutica medicamentosa é a melhor forma de reduzir risco de contágio dos comunicantes. Concluindo, pode ser ressaltada ainda a importância do desenvolvimento e da implementação de estratégia de intervenção visando à melhoria da adesão, permitindo o monitoramento para a melhoria contínua da qualidade da assistência em saúde, especialmente a farmacêutica.

\section{AGRADECIMENTOS}

Os autores agradecem ao Setor de Tuberculose da Prefeitura Municipal de Santa Maria pelas importantes informações fornecidas, e ao Centro Universitário Franciscano pelo financiamento da pesquisa.

\section{REFERÊNCIAS}

1. FIOCRUZ. Fundação Oswaldo Cruz. Escola Nacional de Saúde Pública Sérgio Arouca. Educação a Distância. Controle da tuberculose: uma proposta de integração ensino-serviço. Educação a Distância; coordenado por Maria José Procópio. Rio de Janeiro: EAD/ENSP, 2008.

2. Hijjar MA, Oliveira MJP, Teixeira GM. A tuberculose no Brasil e no mundo. Bol. Pneum. Sanit. 2001; 9(2), jul-dez.

3. Hijjar MA, Gerhardt G, Teixeira GM, Procópio MJ. Retrospecto de controle da tuberculose no Brasil. Rev. de Saúde Pública. 2007; 1(41):50-57.

4. BRASIL. Ministério da Saúde. Departamento de Vigilância Epidemiológica. Manual de recomendações para o controle da tuberculose no Brasil. Brasília: Ministério da Saúde; 2011.

5. Ruffino-Neto A. Programa de Controle da Tuberculose no Brasil. Informe Epidemiológico do SUS. 2001; 10(3):129-138.

6. Duarte R, Carvalho A, Ferreira D, Saleiro S, Lima R, Mota $M$ et al . Abordagem terapêutica da tuberculose e resolução de alguns problemas associados à medicação. Rev Port Pneum. 2010; 16(4): 559-572.

7. Santos Jr. JDO, Pereira BB. Estudo dinâmico da mortalidade por tuberculose no Estado de São Paulo, Brasil: uma abordagem bayesiana. Cad Saúde Pública. 2011; 27(7):1415-1422.
8. Mendes AM, Fensterseifer LM. Tuberculose: porque os pacientes abandonam o tratamento? Bol Pneum Sanit. 2004; 12(1):25-36.

9. Oliveira LGD, Natal S, Camacho LAB. O programa de controle da tuberculose em unidades prisionais de dois estados brasileiros. Cad Saúde Colet. 2012; 20(2):250-257.

10. Andrade RLP, Villa TCS, Pillon S. A influência do alcoolismo no prognóstico e tratamento da tuberculose. Rev Eletr SMAD. 2005; 1(1): 1-8.

11. Nogueira JA, Netto AR, Monroe AA, Gonzales RIC, Villa TCR. Busca ativa de sintomáticos respiratórios no controle da tuberculose na percepção do Agente Comunitário de Saúde. Rev Eletr Enferm. 2007; 9(1):116-118.

12. Muniz JN, Palha PF, Monroe AA, Gonzales RC, Ruffino Netto A, Villa TCS. A incorporação da busca ativa de sintomáticos respiratórios para o controle da tuberculose na prática do agente comunitário de saúde. Ciência e Saúde Coletiva. 2005; 10(2):315-321.

13. Cardoso GCP, Cruz MM, Abreu DMF, Decotelli PV, Chrispim PM, Borenstein JS, et al. A conformidade das ações do tratamento diretamente observado para tuberculose na perspectiva dos profissionais de duas unidades de saúde da cidade do Rio de Janeiro. Cad. Saúde Colet. 2012; 20(2): 203-210. 
14. Cantalice Filho JP. Efeito do incentivo alimentício sobre o desfecho do tratamento de pacientes com tuberculose em uma unidade primária de saúde no município de Duque de Caxias, Rio de Janeiro. J Bras Pneum. 2009; 35(10):992-997.

15. Gonzales RIC, Monroe AA, Assis EG, Palha PF, Villa TCS, Ruffino Netto A. Desempenho de serviços de saúde no Tratamento Diretamente Observado no domicílio para controle da tuberculose. Rev. Esc. Enferm USP. 2008; 42(4): 628-634.

16. Maciel ELN, Silva AP, Meireles W, Fiorotti K, Hadad DJ, Dietze R,. Tratamento supervisionado em pacientes portadores de tuberculose utilizando supervisores domiciliares em Vitória, Brasil. J. Bras. Pneum. 2008; 34(7): 506-513.

17. Cavalcanti MLT, Carvalho RMG, Santos MLF, Sucupira ED, Pessanha GF, Medeiros DA et al. Processos de registro e gerenciamento concernentes aos sistemas de informação da tuberculose nos municípios do estado do Rio de Janeiro prioritários segundo o Fundo Global Tuberculose Brasil, 2009/2010. Cad.de Saúde Colet. 2012; 20(2): 161-168.

18. CEVS. Centro Estadual de Vigilância em Saúde. Secretaria Estadual de Saúde do Rio Grande do Sul. Divisão de Vigilância Sanitária. Nota Técnica Conjunta $n^{\circ}$ 02/2011 - CEVS/SES/RS, CGVS/SMS/POA, IPB-LACEN/RS, 2011.

19. Calver A, Falmer A, Murray M, Strauss O, Streicher E, Hanekom $\mathrm{M}$ et al. Emergence of Increased Resistance and Extensively Drug-Resistant Tuberculosis Despite Treatment Adherence, South Africa. US National Library of Medicine National Institutes of Health. 2010; 16(2): 264-71.

20. Toman K. Tuberculosis, detección de casos y quimioterapia. Preguntas y respuestas. Washington DC: Organización Panamericana de la Salud; 1980.

21. SINAN. Sistema de Informação de Agravos de Notificação. 2013. Disponível em http://dtr2004.saude.gov.br/ sinanweb/. Acesso em 12 de junho de 2013.

22. Brasil. Ministério da Saúde; Secretaria de Vigilância em Saúde. Programa Nacional de Controle da Tuberculose. Manual de recomendações para o controle da tuberculose no Brasil [Internet]. Brasília; 2010 Disponível em:http://portal.saude.gov.br/portal/arquivos/ pdf/manual_de_recomendacoes_controle_tb_novo. Acesso em 14 de junho de 2013

23. World Health Organization (WHO). Multidrug and extensively drug-resistant TB (M/XDR TB): global report on surveillance and response. WHO Report 2010. Geneva; 2010.
24. FIOCRUZ. Fundação Oswaldo Cruz. Glossário de doenças: Tuberculose, ago. 2006. Disponível em http://www.fiocruz.br/ccs/cgi/cgilua.exe/sys/start. htm?infoid $=309 \&$ sid $=6$. Acessado em $<15$ de junho de 2013>.

25. Braga JU, Pinheiro JS, Matsuda JS, Barreto JAP, Feijão AMM. Fatores associados ao abandono do tratamento da tuberculose nos serviços de atenção básica em dois municípios brasileiros, Manaus e Fortaleza, 2006 a 2008. Cad. Saúde Colet. 2012; 20(2): 225-233.

26. Sá LD, Souza KMJ, Nunes MG, Palha PF, Nogueira JÁ, Villa TCS. Tratamento da Tuberculose em Unidades de Saúde da Família: Histórias de Abandono. Texto Contexto Enferm. 2007; 16(4)712-718.

27. Jmachoki MI, Kredo T, Volmink J. Patient education and counselling for promoting adherence to treatment for tuberculosis. Cochrane Database Syst Rev 2012, Issue 5. Art. No.: CD006591.

28. Garfein R, Collins K, Munoz F, Moser K, CerecerCallu P, Sullivan M, et al. High Tuberculosis Treatment Adherence Obtained Using Mobile Phones for Video Directly Observed Therapy: Results of a Binational Pilot Study. J Mobile Technol Med. 2012; 1(4S):30.

29. Finlay A, Lancaster J, Holtz T, Weyer K, Miranda A, Walt MVD. Patient - and provider - level risk factors associated with default from tuberculosis treatment, South Africa, 2002: a case-control study. BMC Public Health. 2012; 12:56.

30. Mesfin M, Newell J, Walley J, Gessessew A, Tesfaye T, Lemma $\mathrm{F}$, et al. Quality of tuberculosis care and its association with patient adherence to treatment in eight Ethiopian districts. Health Policy and Planning. 2009; 24(6):457-466.

31. Boogaard JVD, Msoka E, Homfray M, Kibiki G, Heldens J, Felling A, et al. Exploration of Patient Perceptions of Adherence to Tuberculosis Treatment in Tanzania. Sage Journals. 2012; 22(1): 835-845.

32. Nogueira-Martins MCF, Bógus CM. Considerações sobre a metodologia qualitativa como recurso para o estudo das ações de humanização em saúde. Saúde e Sociedade. 2004; 13(3):44-57.

33. Benevides R, Passos E. A humanização como dimensão pública das políticas de saúde. Ciência e Saúde Colet 2005; 10(3): 561-571. 